Brit. Heart F., 1968, 30, 132.

CASE REPORTS

\title{
Tuberous Sclerosis Presenting as Cardiac Arrhythmia
}

\author{
THOMAS R. TAYLOR
}

From the Division of Medicine, Victoria Infirmary, Glasgow S.2

Tuberous sclerosis is a rare disease of protean manifestations involving primarily the skin, the nervous system, and more rarely a number of other organs, including the kidneys and lungs. The occurrence of rhabdomyomata in the myocardium is fairly common (Crome, 1954). It has been defined as one of the congenital ectodermoses, which include von Recklinghausen's disease, or neurofibromatosis, and Lindau-von Hippel's disease. It is more common in men than in women, and according to Dawson (1954) occurs in about $1 / 300,000$ of the general population and in about $1 / 300$ admissions to epileptic institutions or mental deficiency hospitals. Until 1951 only 400 cases had been reported, and it is, therefore, a relatively uncommon disease.

Most cases reported have been in children and the mortality rate has been very high (De la Cruz and LaVeck, 1962). The age of onset is variable, but 75 per cent of patients are dead before their 20th year. Death is usually due to intercurrent infection, cachexia, status epilepticus, or congestive cardiac failure. The disease is, however, compatible with a long life, especially in milder cases.

The patient described here was an adult in relatively good health who presented with a cardiac arrhythmia.

\section{Case Report}

A 23-year-old Irish labourer was first seen at the Cardiac Department of the Victoria Infirmary, Glasgow, in December 1963, with a four-month history of attacks of dizziness accompanied by breathlessness and palpitation. These attacks occurred apparently spontaneously about twice a month, and lasted up to half an hour. They were often associated with chest tightness, which eased with rest. A year previously he had a solitary episode of haemoptysis, which was associated with a chest cold and had not recurred. There was no history of ankle oedema, skin rash, or seizures at this time.
On this first occasion, clinical examination was negative apart from a cardiac irregularity. The heart was not enlarged and there were no murmurs. An electrocardiogram (Fig. 1) showed numerous multifocal ventricular extrasystoles and a tendency to intraventricular conduction defect. Chest $x$-ray film was negative. There was no facial rash. A provisional diagnosis of cardiomyopathy or coronary artery disease was made. He was treated with quinidine and when seen again a week later the arrhythmia was unchanged. Contact with the patient was then lost because he left the country.

He was seen again at the same clinic in May 1965. In the interval he had been in reasonable health but had a recurrence of his breathlessness a few weeks previously. The dyspnoea was paroxysmal and was associated with episodes of palpitation occurring at rest. A papulovesicular eruption affected the supraorbital region, upper eyelids, nose, and cheeks (Fig. 2), with a large number of papules on each forearm. Dr. R. W. Carslaw diagnosed the eruption as adenoma sebaceum and noted subungual fibromata on both hands, café-au-lait patches on the forearm, and small papillomata on the buccal mucosa: all of these features are known to be associated with tuberous sclerosis.

The heart was not enlarged and the heart sounds were pure; but a complex arrhythmia was seen on the cardiogram with multifocal extrasystoles and, on this occasion, left bundle-branch block. The possibility of rhabdomyoma of the heart was raised as an explanation of the arrhythmia. A presumptive diagnosis of tuberous sclerosis was made and the patient was admitted for investigation, including angiography, in the expectation of demonstrating rhabdomyomatous tumours in the myocardium. Investigations performed initially included full blood count, differential leucocyte count, Wassermann reaction, lupus erythematosus test, serum electrolytes, serum cholesterol, and liver function tests, all of which were normal.

Angiography was performed and the report stated: "The catheter was inserted and placed in the outflow tract of the right ventricle. Frequent ventricular extrasystoles were noted at all stages. Ventricular tachy132 


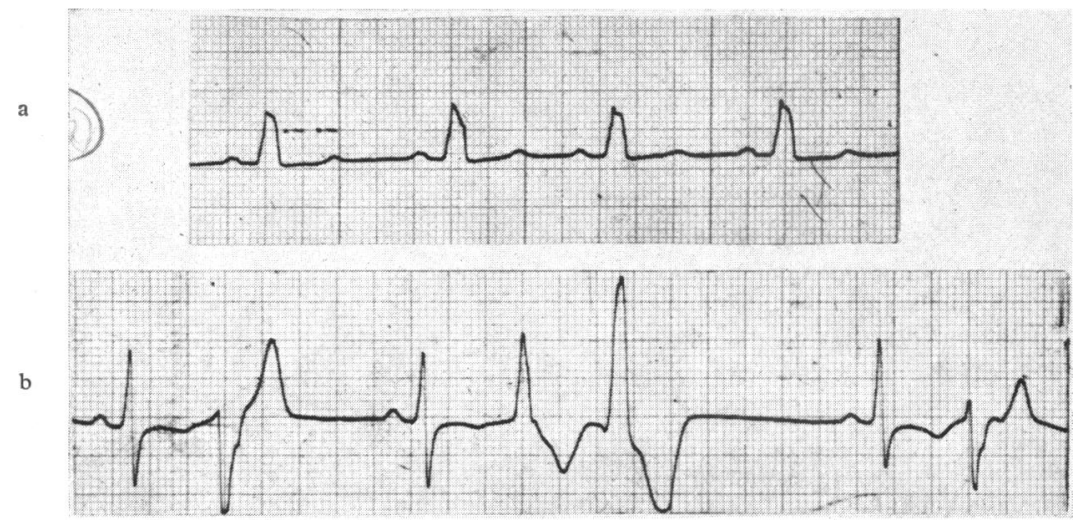
FIG. 1.-(a) Lead I showing tendency to intraventricular conduction defect. (b) Lead V6 showing multi-
focal ventricular extrasystoles.

tract. It was decided that right atrial injection would be the safer measure and this was carried out uneventfully." No myocardial tumour was demonstrated.

The patient's pulse continued to be irregular and he was started on quinidine sulphate. $\mathrm{He}$ showed no response to doses of up to $2.0 \mathrm{~g}$. (30 grains) a day over a period of 12 days and, in fact, became very agitated and upset. Procaine amide was tried for 7 days but this too had to be stopped because of agitation and excitement. Propranolol $10 \mathrm{mg} .4$ times a day was later started with little influence on his arrhythmia. He had

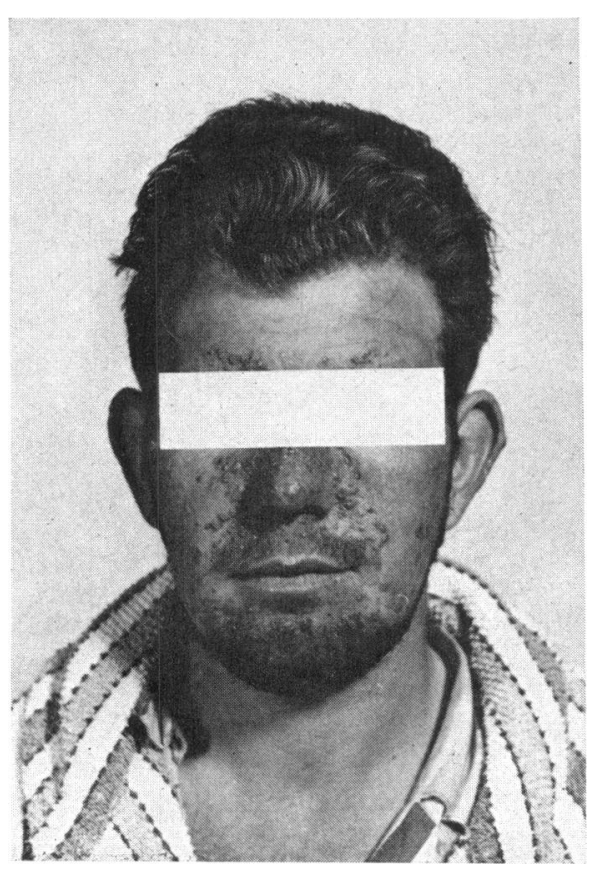

FIG. 2.-Typical rash of adenoma sebaceum. occasional bouts of left arm pain associated with palpitation, and propranolol was eventually stopped.

A number of other investigations were performed to exclude multi-system involvement which is so often found in tuberous sclerosis. An intravenous pyelogram was normal. $X$-ray films of chest, skull, and skeleton, revealed no evidence of intracranial calcification or bony sclerosis. Many patients with tuberous sclerosis are mentally defective. On testing, he had an IQ of 66, which is in the defective range. His verbal IQ was 61 and his performance IQ 76. Electroencephalogram was normal. The only other significant abnormality in the investigation was an abnormal glucose tolerance curve of the "lag curve" or "oxyhyperglycaemic type", which suggested a delayed utilization of glucose. A normal serum galactose excluded glycogen storage disease.

The patient improved despite his persistent arrhythmia and was discharged, whereupon he returned immediately to Ireland. He was seen at the hospital on one occasion 6 months later and felt well, though his pulse remained irregular and the cardiogram still showed multifocal extrasystoles and left bundle-branch block. As far as is known, he has resumed his normal work as a labourer.

\section{Discussion}

The above patient shows several features of classical tuberous sclerosis; namely, mental deficiency, typical tuberous sclerosis rash with papillomatosis, and café-au-lait spots on the skin of the limbs. He had on the other hand no evidence of epilepsy, intracranial calcification, sclerosis of the long bones, or renal neoplasm (which are often seen in this condition). Haemoptysis has already been noted in a series of pulmonary forms of tuberous sclerosis (Dawson, 1954). The abnormal glucose tolerance test is extremely unusual in this condition. The only other case of tuberous sclerosis in which such a finding was reported was shown to 
have an insulinoma of the pancreas (Gutman and Leffkowitz, 1959).

The primary interest in this case, however, is the cardiac arrhythmia. Crome (1954) suggested that rhabdomyomata produced their effects by mechanical interference with heart function or with its conducting mechanism. The multifocal extrasystoles and left bundle-branch block in this case would fit this theory. Rhabdomyomatous nodules are known to occur singly or in small groups in the subendocardial layer or within fibrotic areas in the myocardium. Four cases of fibro-elastosis of the myocardium have been noted in tuberous sclerosis (Crome, 1954). There is, however, only one report of an adult with tuberous sclerosis presenting with a cardiac arrhythmia. Duras (1945) described a man aged 24 with typical tuberous sclerosis who was mentally defective, had a marked behaviour disorder, and who had been incontinent of urine until the age of 14. He suffered from epilepsy and hypersomnia and had an irregular pulse with mild cardiac enlargement and multifocal extrasystoles on electrocardiogram.

A computer-based literature retrieval search was made by the National Library of Medicine in Maryland. No case was found in the world literature in the past 3 years, in which the association of a cardiac arrhythmia and tuberous sclerosis had been noted. This search involved 385,000 articles in 2500 journals in 28 languages.

The relation between rhabdomyomata and tuberous sclerosis is not entirely clear but since over 50 per cent of rhabdomyomata occur in patients with overt tuberous sclerosis (Griffiths, 1965), it seems possible that the remaining cases are formes frustes of the same disease.

\section{Summary}

A case of tuberous sclerosis occurring in a young man with mental deficiency, typical rash, and cardiac arrhythmia has been described. The presentation with an arrhythmia is extremely rare having been reported only once before.

I wish to acknowledge my thanks to Dr. A. A. F. Peel, Dr. R. W. Carslaw, Dr. T. Semple, and Mr H. C. Gray and staff of the Department of Medical Illustrations, Victoria Infirmary.

\section{References}

Crome, L. (1954). The structural features of epiloia, with special reference to endocardial fibroelastosis. $\mathcal{F}$. clin. Path., 7, 137.

Dawson, J. (1954). Pulmonary tuberous sclerosis. Quart.F. Med., 23, 113.

De la Cruz, F. F., and LaVeck, G. D. (1962). Tuberous sclerosis: A review and report of eight cases. Amer. $\mathcal{F}$. ment. Defic., 67, 369.

Duras, F. P. (1945). Cardiac manifestations in a case of tuberous sclerosis. Brit. Heart F., 7, 37.

Griffiths, G. C. (1965). A review of primary tumors of the heart. Progr. cardiocasc. Dis., 7, 465.

Gutman, A., and Leffkowitz, M. (1959). Tuberous sclerosis associated with spontaneous hypoglycæmia. Brit. med. f., 2, 1065. 